\title{
Long non-coding RNA FAM83H-AS1 acts as a potential oncogenic driver in human ovarian cancer
}

\author{
Xiaolei Yuan', Ying Huang ${ }^{2}$, Man Guo ${ }^{1}$, Xiaowei $\mathrm{Hu}^{3}$ and Peiling $\mathrm{Li}^{1 *}$
}

\begin{abstract}
Objective: Ovarian cancer $(\mathrm{OC})$ is one of the most aggressive women cancers with increasing incidence and mortality rates worldwide. Long non-coding RNAs (IncRNAs) could as major players in OC process. Although FAM83H antisense RNA1 (FAM83H-AS1) is demonstrated play an important roles in a many cancers, the detailed function and mechanism has not been reported in OC.

Results: We integrated multiple kinds of bioinformatics approaches and experiments validated method to evaluate functions of FAM83H-AS1 in OC. Some differential expressed IncRNAs were identified between OC and normal control tissues. FAM83H-AS1 was one of most differentially expressed IncRNAs and up-regulated in multiple cancer types. Specially, expression of FAM83H-AS1 was higher in OC and showed difference in diverse stages. High FAM83H-AS1 expression is associated with worse pan-cancer and OC outcomes. FAM83H-AS1-centric network including IncRNA-miRNA, IncRNA-protein and IncRNA-mRNA ceRNA network were constructed to infer the function and mechanism of FAM83H-AS1. There were two methylation sites including cg01399317 and cg20519035 located at FAM83H-AS1. The methylation level of cg01399317 was correlated with gene expression of FAM83H-AS1. The expression level of FAM83H-AS1 was correlated with infiltration level of immune cell including macrophage, neutrphil and dendritic cell in OC patients. Lastly, QRT-PCR showed that the expression of FAM83H-AS1 was higher in OC tissues than normal control tissues.
\end{abstract}

Conclusion: Collectively, these results indicated that FAM83H-AS1 may act as an oncogenic driver and it may be a potential therapy target in OC.

Keywords: Ovarian cancer, IncRNA FAM83H-AS1, Network, Survival, Immune

\section{Background}

In the female reproductive system, ovarian cancer (OC) is one of the most malignant tumors [1]. As cancer statistics in China and United States suggested that the mortality rate of $\mathrm{OC}$ has been rising for the past few years $[2,3]$. there will be approximately 22,240 new cases of ovarian cancer diagnosed and 14,070 ovarian cancer deaths in the United States [4]. OC accounts for

\footnotetext{
*Correspondence: samanly@126.com; peiley@126.com

'Department of Obstetrics and Gynecology, The Second Affiliated Hospital of Harbin Medical University, 246 Xuefu Road, Nangang District, Harbin 150081, Hei Longjiang Province, China

Full list of author information is available at the end of the article
}

$2.5 \%$ of all female malignant tumors and part of deaths for cancer patients due to poor survival which largely driven by late stage diagnoses [5]. Surgical resection combined with platinum-based and taxane-based chemotherapy is the major and standard approach for advanced-stage ovarian treatment [6]. Although advancing insight about mechanism and treatment of OC has been evolved rapidly in recent year, survival rates have improved only slightly over the past 3 decades. Thus, Improving prevention and early detection based on identifying molecular biomarkers are effective ways to enhance survival for OC patients.

C C The Author(s). 2021 Open Access This article is licensed under a Creative Commons Attribution 4.0 International License, which permits use, sharing, adaptation, distribution and reproduction in any medium or format, as long as you give appropriate credit to the original author(s) and the source, provide a link to the Creative Commons licence, and indicate if changes were made. The images or other third party material in this article are included in the article's Creative Commons licence, unless indicated otherwise in a credit line to the material. If material is not included in the article's Creative Commons licence and your intended use is not permitted by statutory regulation or exceeds the permitted use, you will need to obtain permission directly from the copyright holder. To view a copy of this licence, visit http://creativecommons.org/licenses/by/4.0/ The Creative Commons Public Domain Dedication waiver (http://creativecommons.org/publicdomain/zero/1.0/) applies to the data made available in this article, unless otherwise stated in a credit line to the data. 
Long non-coding RNAs (lncRNAs), which are defined as RNA transcripts of $>200$ nucleotides that are not translated into protein [7]. IncRNAs are a highly versatile class of transcripts that have sparked new lines of research in nearly all fields of the life sciences [8]. More recently, emerging studies have identified lncRNAs as major players in many kinds of cancer processes [9]. Many studies revealed that lncRNA play essential roles in proliferation, migration, and invasion of cancers including OC $[10,11]$. For example, IncRNA LINC00176 was highly expressed in OC tissues as well as in OC cell lines, respectively. Knockdown of lncRNA LINC00176 suppresses OC progression by BCL-mediated downregulation of ceruloplasmin [12]. Li et al. suggested that lncRNA UCA1 was upregulated in cisplatin-resistant patient tissues and cell lines. Knockdown of UCA1 inhibited cell proliferation and promoted the cisplatininduced cell apoptosis in OC cells [13]. The expression of lncRNA ABHD11-AS1 in OC tissues was higher compared to normal ovarian tissue. Overexpression of ABHD11-AS1 promoted OC cell proliferation, invasion and migration, and inhibited apoptosis [14]. Specially, Emerging evidence indicates that lncRNAs participate in crosstalk between tumor and tumor immune microenvironment [15]. The regulatory mechanisms of lncRNAs were multiple and complex such as competing endogenous RNA (ceRNA) in cancer including OC [16].

Recently, a novel lncRNA, FAM83H antisense RNA1 (FAM83H-AS1), demonstrated important roles in a many cancers. Zhang et al. reported that FAM83H-AS1 is associated with clinical progression and modulates cell proliferation, migration, and invasion in bladder cancer [17]. FAM83H-AS1 was found overexpressed in HPV-16 positive cervical cancer cell lines in an HPV-16 E6-dependent manner but independently of p53 regulation [18]. Xu et al. indicated that the expression of FAM83H-AS1 was higher in glioma tissues and cell lines and overexpression of FAM83H-AS1 was associated with poor prognosis of glioma. Specially, FAM83H-AS1 was upregulated in OC [19]. However, underlying mechanisms of FAM83H-AS1 regulating functions in OC have yet to be elucidated.

In present study, differential expressed lncRNAs were identified between $\mathrm{OC}$ and normal ovarian tissues. FAM83H-AS1 was differential expressed in many types of cancers. Specially, the expression of FAM83H-AS1 was higher in $\mathrm{OC}$ and fluctuated among diverse stage of OC. FAM83H-AS1 was also associated with survival in pan-cancer and OC. FAM83H-AS1-centric network including lncRNA-miRNA, IncRNA-protein and lncRNAmRNA ceRNA network were constructed. qRT-PCR showed that FAM83H-AS1 was up-regulated in OC tissues. Overall, our findings indicated that FAM83H-AS1 may mediate the oncogenesis process and can be regarded as a prognostic biomarker in OC.

\section{Results}

Some dysregulated genes and IncRNAs were identified in OC

We identified differential expressed coding genes and lncRNAs between OV tissues and normal control tissues to screen candidate genes. 2419 differential expressed lncRNAs and coding genes were identified based on ttest $(P<0.001)$ for OV patients (Fig. 1a). There were 920 $(38.03 \%)$ up-regulated and 1499 (61.97\%) downregulated for all the differential expressed lncRNAs and coding genes (Fig. 1b). We further focused on dysregulated IncRNAs in OC. There were 64 (22.70\%) and 218 (77.30\%) up and down-regulated lncRNAs in OC tissues compared with normal ovarian tissues (Fig. 1c). The results indicated that some dysregulated lncRNAs maybe play essential roles in OC patients. Specially, differential expression of lncRNA FAM83H-AS1 was extremely significant $(P=1.72 \mathrm{E}-101$, fold change value $=3.781)$. Thus lncRNA FAM83H-AS1 was considered as a candidate biomarker for $\mathrm{OC}$ to perform follow analyses.

\section{FAM83H-AS1 expression is dysregulated in human pan- cancer and $\mathrm{OC}$}

In order to characterize FAM83H-AS1 in OC, we first explore the expression of FAM83H-AS1 other cancers. We downloaded and analyzed TCGA data to assess the expression of FAM83H-AS1 in pan-cancer. The result indicated that expression of FER1L4 in all the cancer types including bladder urothelial carcinoma (BLCA); breast invasive carcinoma (BRCA); cervical squamous cell carcinoma (CESC); cholangiocarcinoma (CHOL); colon adenocarcinoma (COAD); esophageal carcinoma (ESCA); head and neck squamous cell carcinoma (HNSC); lung squamous cell carcinoma (LUSC); liver hepatocellular carcinoma (LIHC); lung adenocarcinoma (LUAD); kidney renal papillary cell carcinoma (KIRP); prostate adenocarcinoma (PRAD); rectum adenocarcinoma (READ); skin cutaneous melanoma (SKCM); stomach adenocarcinoma (STAD); pancreatic adenocarcinoma (PAAD); thymoma, (THYM) and ovarian serous cystadenocarcinoma (OV) was significantly differential compared with their noncancer counterparts (Fig. 2a). And the expression of FAM83H-AS1 was up-regulated in any cancer type. FAM83H-AS1 was also dysregulated in integrated pancancer patients (Fig. 2b). The abnormal expression of FAM83H-AS1 was also observed in OC (Fig. 2c). In addition, we also found that expression level of FAM83HAS1 changed in diverse stages of OC (Fig. 2d).

\section{FAM83H-AS1 was associated with prognosis in pan- cancer and $\mathrm{OC}$}

Kaplan-Meier analysis and the log-rank test were used to explore the association between FAM83H-AS1 expression and survival status in $\mathrm{OC}$ patients in present 
A

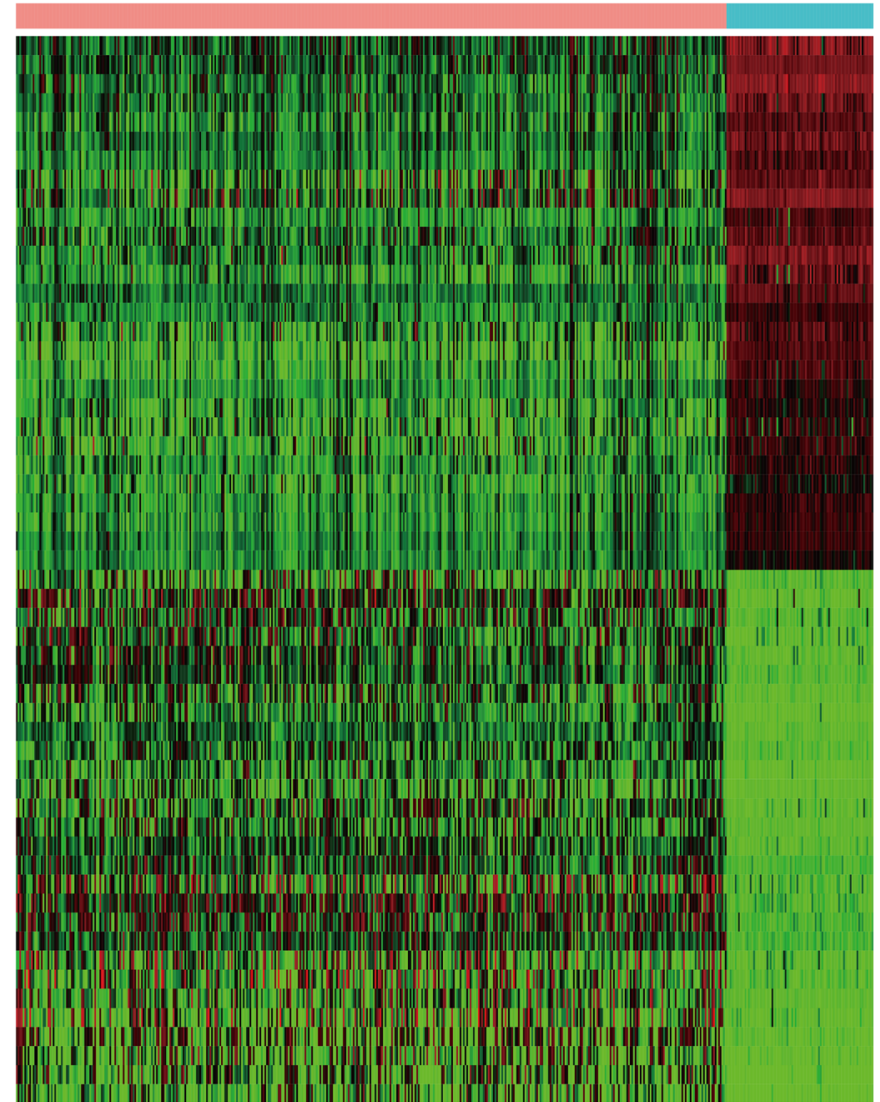

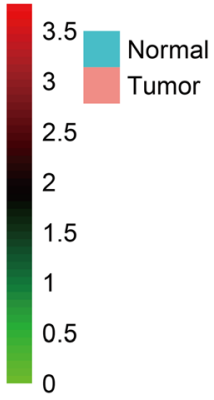

B

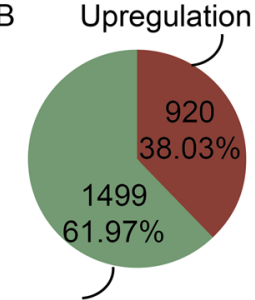

Downregulation

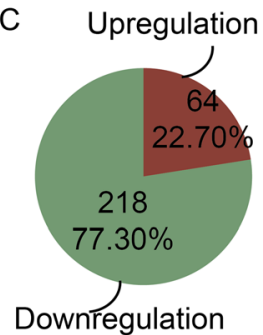

Fig. 1 Some dysregulated genes and IncRNAs were identified in OC. a Heatmap of significantly differentially expressed IncRNAs and coding genes in OC patients. Red indicates genes that had higher expression level and green indicates genes had lower expression. $\mathbf{b}$ The pie chart shows percent of up- and down-regulated IncRNAs and coding genes. c The pie chart shows percent of up- and down-regulated IncRNAs

study. We found higher expression of FAM83H-AS1 was associated with worse overall survival in pan-cancer $(P<0.0001$, Fig. 3a). As except, higher expression of FAM83H-AS1 was also associated with worse overall survival in OC patients $(P=0.044$, Fig. 3b). The results indicated that lncRNA FAM83H-AS1 maybe could as a unfavorable prognostic biomarker for OC patients.

\section{FAM83H-AS1-centric IncRNA-miRNA, IncRNA-protein and IncRNA-mRNA ceRNA networks could show the special function of FAM83H-AS1}

We try to explore the function of FAM83H-AS1 based on studying its interacted miRNA and coding genes using multiple networks. First, a lncRNA-mRNA network was constructed, which contained 22 interacted miRNAs (Fig. 4a). The co-expression of FAM83H-AS1 and any interacted miRNA were also calculated. The expression of miR-211 was significantly positive correlated with FAM83H-AS1 in OC patients (Fig. 4b). Present study demonstrated that the expression of miR-211 is differential expressed in $\mathrm{OC}$ tissues and cell lines compared to normal epithelial ovarian tissue and human ovarian surface epithelial cells, respectively. miR-211 was also found to arrest cells in the G0/G1-phase, inhibit proliferation and induce apoptosis [20]. Second, a lncRNA-protein (or coding gene) network was constructed (Fig. 4c). There were three coding genes could interacted with FAM83HAS1. These three coding genes all play essential roles in cancers [21-23]. At last, we also constructed a lncRNAmRNA ceRNA network (Fig. 4d). TP53 was a ceRNA of FAM83H-AS1 and mutations in the TP53 gene are still by far the most frequent genomic event in cancer genomes [24]. All the results indicated that FAM83H-AS1 maybe play its role by interacting with some OC-related coding genes and miRNAs.

\section{Expression level of FAM83H-AS1 was associated with methylation and immune}

We found there were two methylation sites including cg01399317 and cg20519035 located at FAM83H-AS1 (Fig. 5a). The methylation level of cg01399317 was correlated with gene expression of FAM83H-AS1 $(P=$ 0.011 , Fig. 5b). Recent years, immunotherapy have resulted in clinical success in treating late-stage cancers 


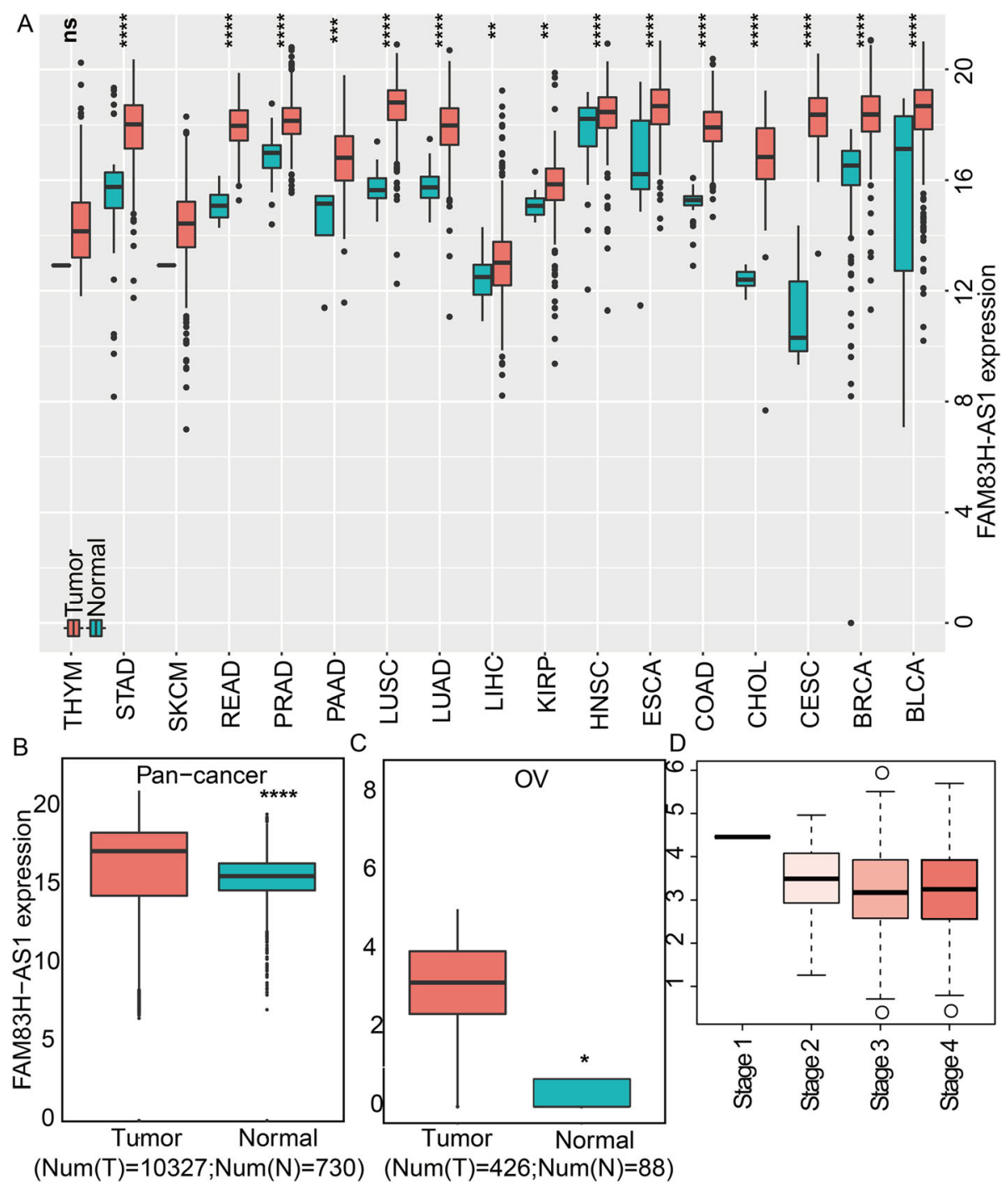

Fig. 2 FAM83H-AS1 expression is upregulated in human pan-cancer and OC. a The box plots shows the expression of FAM84H-AS1 in multiple cancer types. Red and blue represent tumor and normal samples. b The expression of FAM84H-AS1 in pan-cancer. c The expression of FAM84HAS1 in OC. $\mathbf{d}$ The expression of FAM84H-AS1 in diverse stages of OC

[25]. Some immune signature genes have also been identified and could be applied to characterize immune infiltrates and predict clinical outcome for cancers. We also explored the associations between immune and FAM83HAS1. The expression level of FAM83H-AS1 was associated with infiltration level of immune cell including macrophage $(P=1.34 \mathrm{e}-9)$, neutrphil $(P=7.93 \mathrm{e}-03)$ and dendritic cell $(P=2.33 \mathrm{e}-03)$ in OC patients (Fig. $5 \mathrm{c})$. The results indicated that FAM83H-AS1 maybe could be used to characterize immune infiltrates for OC patients.

\section{FAM83H-AS1 was up-regulated in OC tissues by qRT-PCR} validation

FAM83H-AS1 levels was significantly differentially expressed in $\mathrm{OC}$ tissues compared with corresponding adjacent non-tumor tissues $(P=0.045$, Fig. 6a). The foldchange value of FAM83H-AS1 was 2.9 in OC and control tissues. The expression level of FAM83H-AS1 was significantly higher in OC tissues than control tissues (Fig. 6b).

\section{Discussion}

Multiple lines of evidence suggested that non-coding RNAs are majority of genome transcripts, however, only a small part of them have been characterized to be biologically functional [26]. In recent years, more and more studies focused on implicating the role of IncRNAs in diseases including cancers [27-29]. Some studies had reproted that IncRNA FAM83H-AS1 was associated with many types of cancers such as breast cancer [30], colorectal cancer [31] and colon cancer [32]. However, 

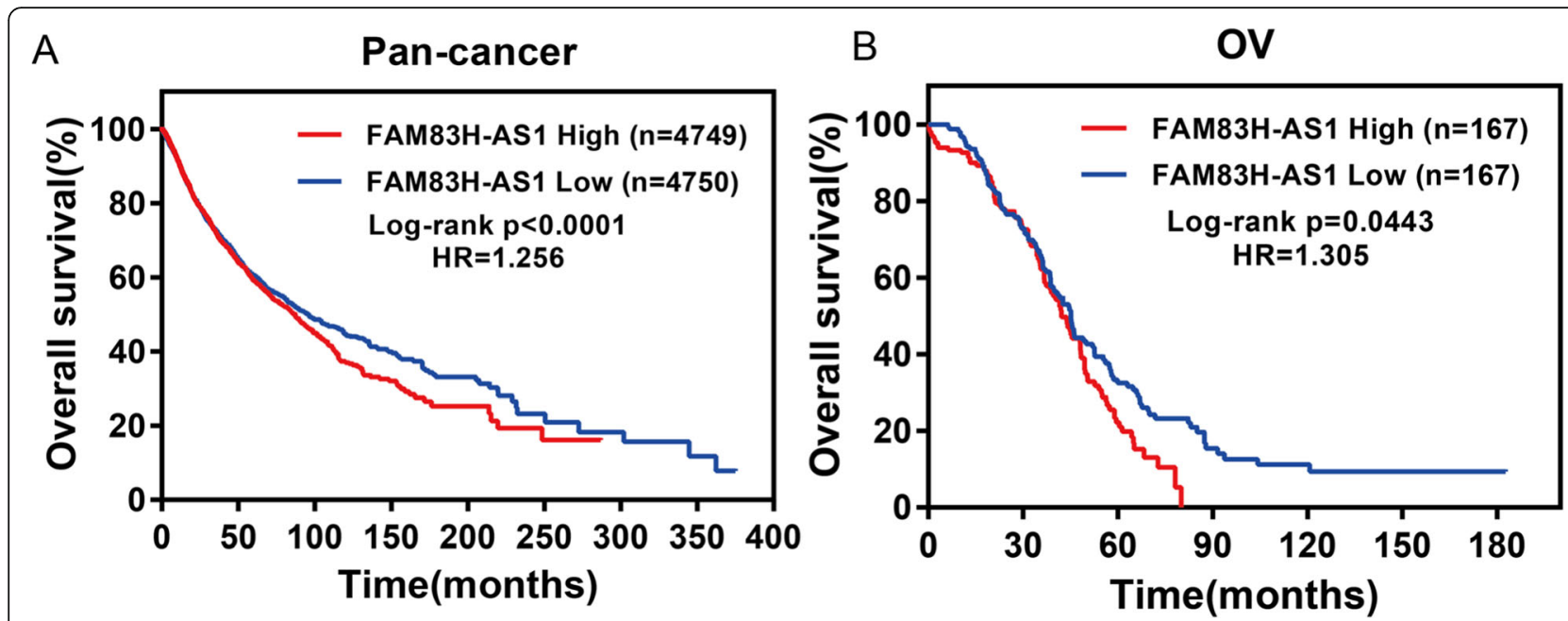

Fig. 3 Kaplan-Meier survival curves for FAM83H-AS1 associated with overall survival. a High expression of FAM83H-AS1 correlates with worse OS in pan-cancer patients and (b) OC patients. X- and Y-axis represent survival time and overall survival. Red and blue lines represent high and low expression of FAM83H-AS1

the function and mechanism of FAM83H-AS1 in OC has not been systemically studied.

In present study, we found FAM83H-AS1 as a novel and essential lncRNA involved in OC. We showed that FAM83H-AS1 was significantly upregulated in many kinds of cancer tissues compared with control normal tissues. In OC patients, FAM83H-AS1 was one of top differential expressed lncRNAs. Specially, expression of FAM83H-AS1 also showed difference in diverse stage of OC. Higher expression of FAM83H-AS1 was associated with worse overall survival in pan-cancer and OC. All above results indicated that FAM83H-AS1 may act as an oncogenic driver in OC.

In order to further explore the biological function and mechanism of FAM83H-AS1, some regulatory networks were constructed. Previous studies suggested that interacted or co-expressed coding genes could represented the function of lncRNAs [33]. Thus, we try to use the function of coding genes and miRNAs to estimate the role of FAM83H-AS1 in OC. In present study, the expression of miR-211 was significantly positive correlated with FAM83H-AS1 in OC patients. The role of miR-211 in diagnosis, prognosis and treatment in $\mathrm{OC}$ had been reported in many studies. For example, wang et al. found that miR-211 inhibited most of DNA damage responserelated genes, and proposed that miR-211 might affect the sensitivity of OC cells to platinum by targeting multiple DNA damage response-related genes and thereby determine the prognosis of OC [34]. In addition, miR211 could sponge lncRNA MALAT1 to suppress tumor growth and progression through inhibiting PHF19 in OC [35]. We could infer that FAM83H-AS1 maybe also play a key role in $\mathrm{OC}$ based on above results.

Many evidences taken together suggested that OC patients could potentially benefit from immunotherapy
[36]. Identifying immune-related targets could provide assistance for immunotherapy of OC patients. As far as we know, the immune function of FAM83H-AS1 had not been revealed in OC. In our study, we also explore the relationships between immune and FAM83H-AS1. The expression level of FAM83H-AS1 was associated with infiltration level of immune cell including macrophage, neutrphil and dendritic cell in OC patients. The result indicated that FAM83H-AS1 had potential to become a immune-related signature in OC. Our method identified novel candidates associated with OC development and prognosis, which require further research and experimental validation.

\section{Conclusions}

The present study clarified the role of lncRNA FAM83HAS1 in OC patients. It also demonstrated that the associations between immune and FAM83H-AS1 in OC. The analyses are helpful to identify specific biomarker and drug repurposing candidates for OC. In summary, the present study expands the oncogenic lncRNA landscape of OC and reveals that IncRNA FAM83H-AS1 may act as a potential therapy target for OC.

\section{Materials and methods}

Obtain and procession of public pan-cancer and OC data We obtained lncRNA, miRNA, gene expression and methylation (level 3) data, as well as clinical data of all cancer types including OC, from The Cancer Genome Atlas (TCGA, Release: 2017-09-08, https://portal.gdc.cancer.gov/). The gene expression data of normal tissues for ovarian was obtained from GTEX portal (https://www.gtexportal.org/home/index. $\mathrm{html})$. Patients in all the cancer types were integrated as pancancer patients. The sample numbers in each cancer type 


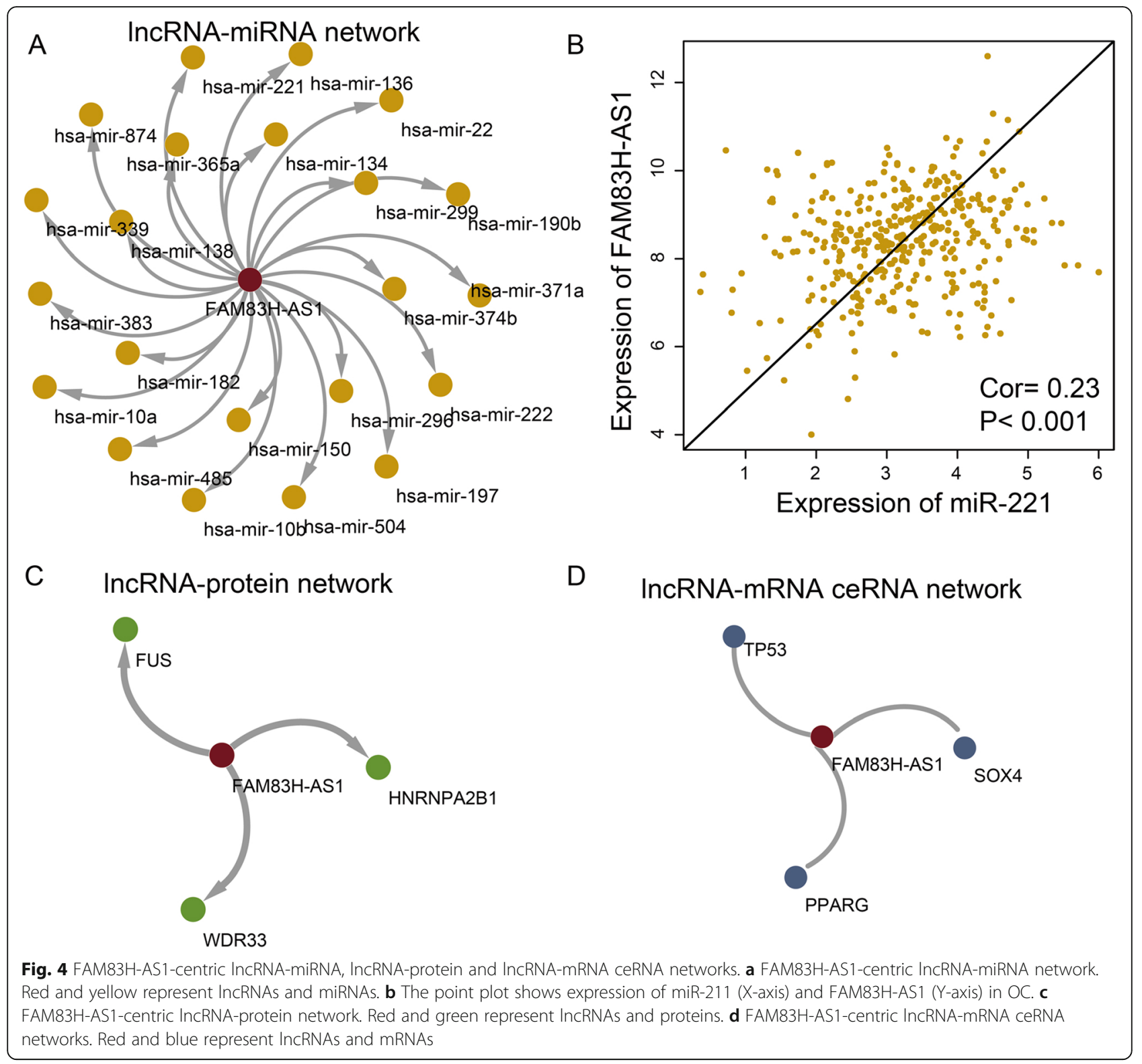

were shown in Table S1. To filter gene, miRNA, and lncRNA not expressed across all samples, the items with expression values of 0 in all of the samples were excluded. Any remaining expression values of 0 were set to the minimum value of all samples, and all values were $\log 2$-transformed. Lastly, 14,619 (96.70\%) IncRNAs were retained for subsequent analysis. Expression of FAM83H-AS1 was dichotomized using median expression as the cutoff to define "high value" at or above the median versus "low value" below the median.

\section{Construction of IncRNA-miRNA, IncRNA-protein and IncRNA-mRNA ceRNA networks for FAM83H-AS1}

In order to describe the functions of FAM83H-AS1, some interacted regulatory networks were constructed. IncRNA-miRNA network was constructed based on experimentally verified associations between miRNAs and lncRNAs were identified in starBase v3.0 (http://starbase.sysu.edu.cn/) [37] and DIANA-LncBase 3.0 (www.microrna.gr/LncBase) [38]. IncRNA-protein data was download from starBase v3.0 and NPInter v2.0 (http://bigdata.ibp.ac.cn/ npinter4) [39] supported by AGO CLIP-seq data. In order to build the IncRNA-mRNA ceRNA network, a hypergeometric test was used to evaluate whether the two lncRNAs have a potential ceRNA relationship by considering their shared interactive miRNAs. $P<.05$ was regarded as statistically significant. All the networks were constructed by Cytoscape 3.3.0 (http://www.cytoscape.org/). 


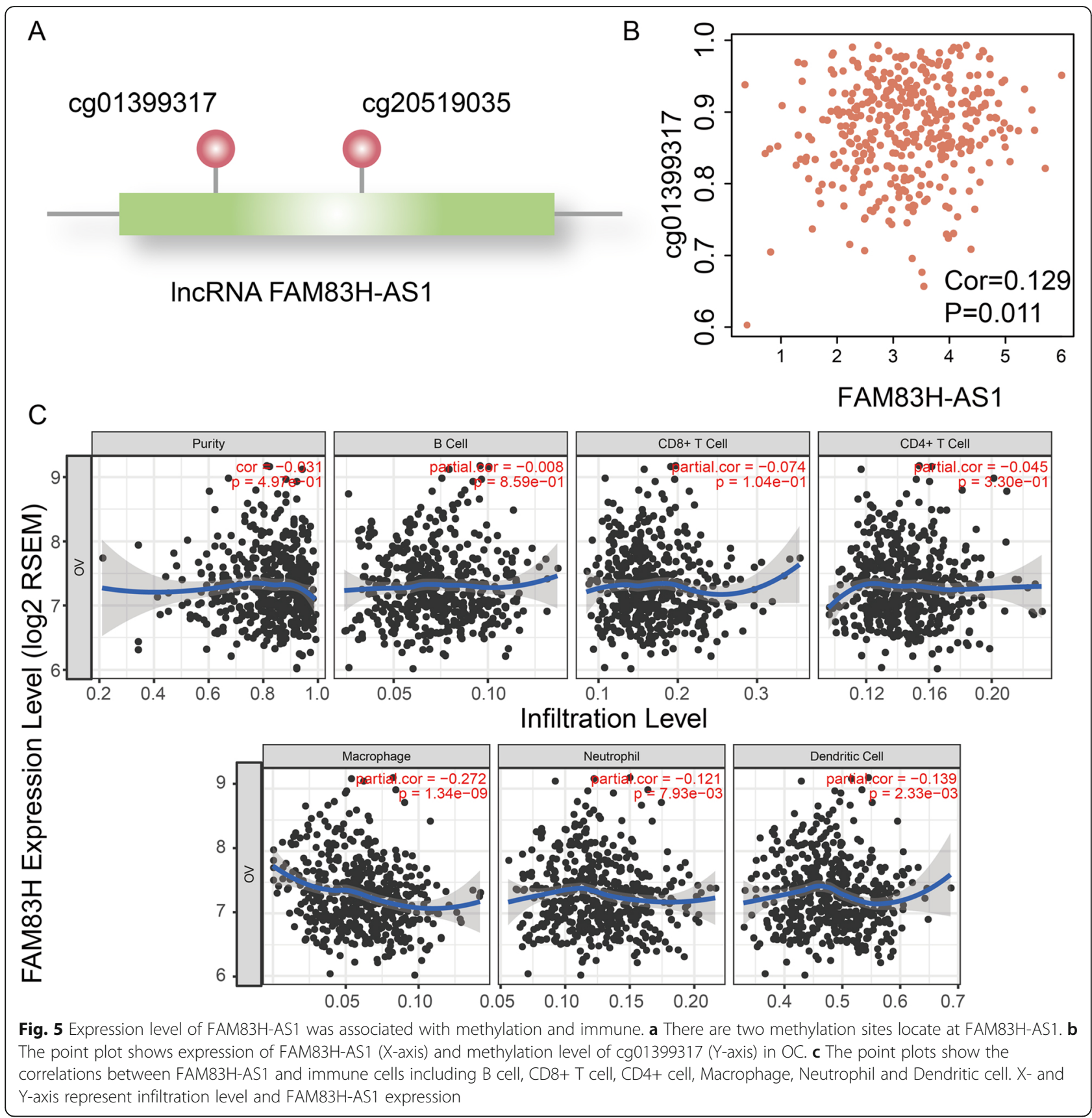

Obtain of DNA methylation profile of OC

DNA methylation profile was measured experimentally using the Illumina Infinium HumanMethylation450 platform. Beta values were derived at the Johns Hopkins University and University of Southern California TCGA genome characterization center (TCGA, Release: 201709-08, https://portal.gdc.cancer.gov/). DNA methylation values, described as beta values, are recorded for each array probe in each sample via BeadStudio software. DNA methylation beta values are continuous variables between 0 and 1 , representing the ratio of the intensity of the methylated bead type to the combined locus intensity. Thus higher beta values represent higher level of DNA methylation and lower beta values represent lower level of DNA methylation. Genomic loacations of methylation site were also obtained from TCGA.

\section{Differential expressed and co-expressed analyses}

T- test was used to calculate the differential expression of all genes and lncRNAs between cancer and normal control samples. $P<.05$ was considered as 

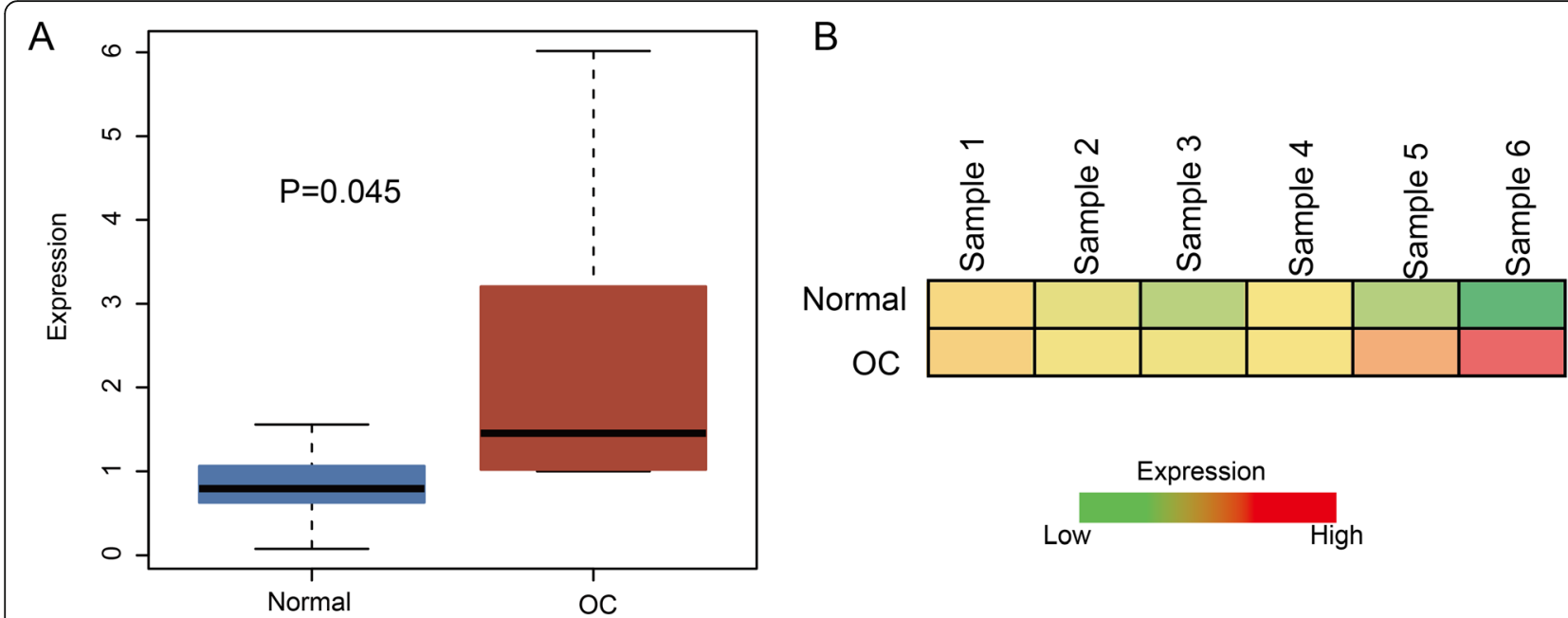

Fig. 6 Expression levels of FAM83H-AS1 in OC by qRT-PCR. a Relative expression levels of FAM83H-AS1 in OC tissue and adjacent noncancerous tissues. $\mathbf{b}$ The heatmap shows the expression of FAM83H-AS1. Red and green represent high and low expression level

significant differential expressed genes and lncRNA. Pearson's correlation coefficients (PCCs) were calculated between FAM83H-AS1 and its interacted miRNAs in lncRNA-miRNA network. In addition, co-expression of methylation level and FAM-83HAS1 expression was also calculated. $P<.05$ was considered as significant co-expressed interactions.

\section{Survival analysis for the FAM83H-AS1 in pan-cancer and OC}

The patients were divided into two groups based on median value of FAM83H-AS1 expression. Kaplan-Meier method and log-rank test were used to evaluate the survival difference in patients with high and low expression of FER1L4. $P<.05$ was regarded as statistically significant.

\section{Tumor-immune infiltrating cells associated with FAM83H-} AS1 in OC

The associations between all tumor-immune infiltrating cells and the FAM83H-AS1 were analyzed via the Tumor Immune Estimation Resource (TIMER) platform (https://cistrome. shinyapps.io/timer/), a web tool for studying tumor-infiltrating immune cells and their interactions with cancer cells [40]. B-cells, CD4 + T-cells, CD8 + T-cells, dendritic cells, macrophages and neutrophils were included in the correlated analyses.

\section{Patients and tissue samples}

OC patients with a histological diagnosis who had undergone surgical resection and had not received chemotherapy or radiotherapy were extracted in our study. Lastly, eight OC tissues and corresponding adjacent normal ovarian tissues were obtained from The Third Affiliated Hospital of Harbin Medical University.
The tissue samples were frozen in liquid nitrogen and stored at $-80^{\circ} \mathrm{C}$ until experiment. The pathological diagnosis were confirmed by three independent senior pathologists. The study was approved by the Research Ethics Committee of The Third Affiliated Hospital of Harbin Medical University. All patients received written informed consent and disposed of specimens in accordance with accepted ethical standards. The clinicopathological features of all patients are indicated in Table 1.

Quantitative real-time reverse transcription PCR (qRT-PCR) Total RNA was extracted from fresh frozen samples and cells using Trizol Reagent (Invitrogen, Carlsbad, CA, USA) according to the manufacturer's instructions. Total RNA $(2 \mu \mathrm{g})$ was reverse-transcribed into cDNA using Transcriptor First Strand cDNA Synthesis Kit (Roche, Vilvoord, Brussel, Belgium). The relative levels of EGOT to glyceraldehyde 3-phosphate dehydrogenase (GAPDH) control transcripts were determined by qPCR using the ABI 7500 Fast RealTime PCR System (Invitrogen). The primer sequences were as follows. FAM83H-AS1: forward 5'-ACTA CAGGCACCCACCACCAC-3', reverse 5'-TGAG ACGGGCGGGATCACAAGG-3'; GAPDH: forward 5'-ACCACAGTCCATGCCATCAC-3', reverse 5' TCCACCCTGTTGCTGTA-3'. The qRT-PCR amplification was performed in triplicate reactions starting at $95^{\circ} \mathrm{C}$ for $10 \mathrm{~min}$, followed by 40 cycles at $95^{\circ} \mathrm{C}$ for $10 \mathrm{~s}$, and $60^{\circ} \mathrm{C}$ for $60 \mathrm{~s}$. Quantitative normalization of EGOT cDNA was performed in each sample using GAPDH expression as an internal control. The relative level of EGOT transcripts to control GAPDH was determined by the $2-\Delta \Delta C T$ method. Each sample was examined in triplicate. 
Table 1 The clinicopathological features of all patients

\begin{tabular}{|c|c|c|c|c|c|c|c|c|c|c|c|c|}
\hline No. & $\begin{array}{l}\text { Age } \\
\text { (year) }\end{array}$ & $\begin{array}{l}\text { Tumor size } \\
(\mathrm{cm})\end{array}$ & $\begin{array}{l}\text { Lymph node } \\
\text { metastasis }\end{array}$ & Stage & Pathological type & Treatment & $\begin{array}{l}\text { ER } \\
\text { positive } \\
\text { cells (\%) }\end{array}$ & $\begin{array}{l}\text { PR } \\
\text { positive } \\
\text { cells (\%) }\end{array}$ & $\begin{array}{l}\text { WT- } \\
1\end{array}$ & P53 & $\begin{array}{l}\text { KI67 } \\
(\%)\end{array}$ & MDR \\
\hline 1 & 53 & $R: 13 \times 13 \times 10$ & No & $\mathrm{IA}$ & $\begin{array}{l}\text { Advanced serous } \\
\text { adenocarcinoma of right } \\
\text { ovary }\end{array}$ & No & 70 & $<5 \%$ & + & $3+$ & 70 & + \\
\hline 2 & 48 & $\begin{array}{l}L: 4.5 \times 3.5 \times 2.0, \\
\text { R: } 7.5 \times 4.5 \times 3.0\end{array}$ & No & $\| B$ & $\begin{array}{l}\text { Advanced serous } \\
\text { adenocarcinoma of bilateral } \\
\text { ovary }\end{array}$ & No & 90 & 40 & - & $3+$ & 60 & - \\
\hline 3 & 43 & $L: 7 \times 6 \times 5, R: 8 \times 6 \times 5$ & Yes & $\| B$ & $\begin{array}{l}\text { Advanced serous } \\
\text { adenocarcinoma of bilateral } \\
\text { ovary }\end{array}$ & No & 70 & 30 & + & $2+$ & 70 & - \\
\hline 4 & 56 & $L: 8 \times 7 \times 7$ & Yes & $\| C$ & $\begin{array}{l}\text { Advanced serous } \\
\text { adenocarcinoma of left } \\
\text { ovary }\end{array}$ & No & 90 & 5 & + & $3+$ & 80 & - \\
\hline 5 & 56 & $L: 8 \times 7 \times 6$ & No & $\mathrm{IA}$ & $\begin{array}{l}\text { Advanced serous } \\
\text { adenocarcinoma of left } \\
\text { ovary }\end{array}$ & No & 70 & - & + & $3+$ & - & - \\
\hline 6 & 53 & $L: 20 \times 16 \times 8$ & No & IC & $\begin{array}{l}\text { Advanced serous } \\
\text { adenocarcinoma of left } \\
\text { ovary }\end{array}$ & No & 50 & 10 & + & + & 30 & - \\
\hline
\end{tabular}

$R$ right ovary, $L$ left ovary

\section{Supplementary Information}

The online version contains supplementary material available at https://doi. org/10.1186/s13048-020-00756-y.

Additional file 1: Table S1. The sample numbers in each cancer type.

\section{Acknowledgments}

Not applicable.

\section{Disclosure of interest}

The authors declare that they have no competing interest.

\section{Patient consent for publication}

Not applicable.

\section{Authors' contributions}

LPL conceived and designed the experiments, YXL, HY, GM and HXW analyzed the data, and YXL wrote the manuscript. The author(s) read and approved the final manuscript.

\section{Funding}

This study was supported by the Heilongjiang Postdoctoral Found to pursue scientific research (LBH-Z172219) and China Postdoctoral Science Foundaction grants (2018M641855).

\section{Availability of data and materials}

The data that support the findings of this study are available from the corresponding author upon reasonable request.

\section{Ethics approval and consent to participate}

Ethical approval was not needed because this is a Bioinformatics analysis.

\section{Consent for publication}

Not applicable.

\section{Competing interests}

The authors declare that they have no competing interests.

\section{Author details}

${ }^{1}$ Department of Obstetrics and Gynecology, The Second Affiliated Hospital of Harbin Medical University, 246 Xuefu Road, Nangang District, Harbin 150081,
Hei Longjiang Province, China. ${ }^{2}$ Harbin Medical University, 157 Baojian Road, Nangang District, Harbin 150081, Hei Longjiang Province, China. ${ }^{3}$ Medical ward 7, Cancer Hospital Affiliated to Harbin Medical University, 150 Haping Road, Nangang District, Harbin 150081, Hei Longjiang Province, China.

Received: 20 July 2020 Accepted: 15 December 2020

Published online: 07 January 2021

References

1. Krzystyniak J, Ceppi L, Dizon DS, Birrer MJ. Epithelial ovarian cancer: the molecular genetics of epithelial ovarian cancer. Ann Oncol. 2016;27(Suppl 1):i4-i10. https://doi.org/10.1093/annonc/mdw083.

2. Chen W, et al. Cancer statistics in China, 2015. CA Cancer J Clin. 2016;66(2): 115-32. https://doi.org/10.3322/caac.21338. Epub 2016 Jan 25.

3. Siegel RL, Miller KD, Jemal A. Cancer statistics, 2015. CA Cancer J Clin. 2015; 65:5.

4. Torre LA, et al. Ovarian cancer statistics, 2018. CA Cancer J Clin. 2018;68(4): 284-96. https://doi.org/10.3322/caac.21456.

5. Ubachs J, et al. Sarcopenia and ovarian cancer survival: a systematic review and meta-analysis. J Cachexia Sarcopenia Muscle. 2019;10:1165.

6. Nick AM, Coleman RL, Ramirez PT, Sood AK. A framework for a personalized surgical approach to ovarian cancer. Nat Rev Clin Oncol. 2015;12:239.

7. Wang KC, Chang HY. Molecular mechanisms of long noncoding RNAs. Mol Cell. 2011:43:904.

8. Grote P, Boon RA. LncRNAs Coming of Age. Circ Res. 2018;123:535.

9. Bach DH, Lee SK. Long noncoding RNAs in cancer cells. Cancer Lett. 2018; 419:152.

10. Worku T, et al. Long non-coding RNAs: the new horizon of gene regulation in ovarian Cancer. Cell Physiol Biochem. 2017;44:948.

11. Fu LL, et al. Role of IncRNAs as novel biomarkers and therapeutic targets in ovarian Cancer. Crit Rev Eukaryot Gene Expr. 2017;27:183.

12. Dai L, Niu J, Feng Y. Knockdown of long non-coding RNA LINC00176 suppresses ovarian cancer progression by BCL3-mediated down-regulation of ceruloplasmin. J Cell Mol Med. 2019.

13. Li Z, et al. IncRNA UCA1 Mediates Resistance to Cisplatin by Regulating the miR-143/FOSL2-Signaling Pathway in Ovarian Cancer. Mol Ther Nucleic Acids. 2019:17:92

14. Wu DD, et al. Role of the IncRNA ABHD11-AS1 in the tumorigenesis and progression of epithelial ovarian cancer through targeted regulation of RhoC. Mol Cancer. 2017;16:138.

15. Zhang Y, Liu Q, Liao Q. Long noncoding RNA: a dazzling dancer in tumor immune microenvironment. J Exp Clin Cancer Res. 2020;39:231. 
16. Braga EA, et al. LncRNAs in Ovarian Cancer Progression, Metastasis, and Main Pathways: ceRNA and Alternative Mechanisms. Int J Mol Sci. 2020;21.

17. Shan $\mathrm{H}$, et al. FAM83H-AS1 is associated with clinical progression and modulates cell proliferation, migration, and invasion in bladder cancer. J Cell Biochem. 2019;120:4687.

18. Barr JA, et al. Long non-coding RNA FAM83H-AS1 is regulated by human papillomavirus 16 E6 independently of p53 in cervical cancer cells. Sci Rep. 2019:9:3662.

19. Dou Q, et al. LncRNA FAM83H-AS1 contributes to the radioresistance, proliferation, and metastasis in ovarian cancer through stabilizing HuR protein. Eur J Pharmacol. 2019:852:134.

20. Xia B, Yang S, Liu T, Lou G. miR-211 suppresses epithelial ovarian cancer proliferation and cell-cycle progression by targeting Cyclin D1 and CDK6. Mol Cancer. 2015;14:57.

21. Murakami T, et al. Effective molecular targeting of CDK4/6 and IGF-1R in a rare FUS-ERG fusion CDKN2A-deletion doxorubicin-resistant Ewing's sarcoma patient-derived orthotopic xenograft (PDOX) nude-mouse model. Oncotarget. 2016;7:47556.

22. Schonemann L, et al. Reconstitution of CPSF active in polyadenylation: recognition of the polyadenylation signal by WDR33. Genes Dev. 2014;28: 2381.

23. Santarosa $M_{1}$, et al. BRCA1 modulates the expression of hnRNPA2B1 and KHSRP. Cell Cycle. 2010;9:4666.

24. Silwal-Pandit L, Langerod A, Borresen-Dale AL. TP53 Mutations in Breast and Ovarian Cancer. Cold Spring Harb Perspect Med. 2017;7.

25. Ribas A, Wolchok JD. Cancer immunotherapy using checkpoint blockade. Science. 2018;359:1350

26. ENCODE Project Consortium. An integrated encyclopediaf DNA elements in the human genome. Nature. 2012;489(7414):57-74. https://doi.org/10.1038/ nature 11247.

27. Fang K, et al. LncRNA ST8SIA6-AS1 promotes proliferation, migration and invasion in breast cancer through the p38 MAPK ssignalling pathway. Carcinogenesis. 2019

28. Wang $X$, et al. A IncRNA coordinates with Ezh2 to inhibit HIF-1alpha transcription and suppress cancer cell adaption to hypoxia. Oncogene. 2019.

29. Yang J, et al. Long noncoding RNA LCAT1 functions as a ceRNA to regulate RAC1 function by sponging miR-4715-5p in lung cancer. Mol Cancer. 2019; 18:171.

30. Yang F, et al. Identification of IncRNA FAM83H-AS1 as a novel prognostic marker in luminal subtype breast cancer. Onco Targets Ther. 2016;9:7039.

31. Lu S, Dong W, Zhao P, Liu Z. IncRNA FAM83H-AS1 is associated with the prognosis of colorectal carcinoma and promotes cell proliferation by targeting the Notch signaling pathway. Oncol Lett. 2018;15:1861.

32. Yang L, Cui J, Wang Y, Tan J. FAM83H-AS1 is upregulated and predicts poor prognosis in colon cancer. Biomed Pharmacother. 2019;118:109342.

33. Zhao Z, et al. Co-LncRNA: investigating the IncRNA combinatorial effects in GO annotations and KEGG pathways based on human RNA-Seq data. Database (Oxford). 2015;2015.

34. Wang $T$, et al. miR-211 facilitates platinum chemosensitivity by blocking the DNA damage response (DDR) in ovarian cancer. Cell Death Dis. 2019;10:495.

35. Tao F, Tian X, Ruan S, Shen M, Zhang Z. miR-211 sponges IncRNA MALAT1 to suppress tumor growth and progression through inhibiting PHF19 in ovarian carcinoma. FASEB J. 2018;fj201800495RR.

36. Ghisoni E, Imbimbo M, Zimmermann S, Valabrega G. Ovarian Cancer Immunotherapy: Turning up the Heat. Int J Mol Sci. 2019;20.

37. Li JH, Liu S, Zhou H, Qu LH, Yang JH. starBase v2.0: decoding miRNA-ceRNA, miRNA-ncRNA and protein-RNA interaction networks from large-scale CLIPSeq data. Nucleic Acids Res. 2014;42:D92.

38. Karagkouni D, et al. DIANA-LncBase v3: indexing experimentally supported miRNA targets on non-coding transcripts. Nucleic Acids Res. 2019.

39. Hao Y, et al. NPInter v3.0: an upgraded database of noncoding RNAassociated interactions. Database (Oxford). 2016;2016.

40. Li B, et al. Comprehensive analyses of tumor immunity: implications for cancer immunotherapy. Genome Biol. 2016;17:174

\section{Publisher's Note}

Springer Nature remains neutral with regard to jurisdictional claims in published maps and institutional affiliations.

\section{Ready to submit your research? Choose BMC and benefit from:}

- fast, convenient online submission

- thorough peer review by experienced researchers in your field

- rapid publication on acceptance

- support for research data, including large and complex data types

- gold Open Access which fosters wider collaboration and increased citations

- maximum visibility for your research: over $100 \mathrm{M}$ website views per year

At BMC, research is always in progress.

Learn more biomedcentral.com/submissions 